\title{
A percepção dos técnicos de enfermagem quanto aos erros no preparo e administração de medicamentos
}

\begin{abstract}
RESUMO
Objetivo: Identificar na percepção do técnico de enfermagem quais fatores contribuem para o ato iatrogênico durante o preparo e administração de medicamentos. Método: Pesquisa de campo de abordagem qualitativa, realizada no setor de clínica médica de um Hospital geral privado do Rio de Janeiro. Por meio de um questionário semiestruturado aplicado a 40 profissionais técnicos de enfermagem. Resultados: Os fatores prevalentes para indução ao erro no preparo e administração de medicamentos mais citados na visão dos profissionais são: dupla jornada, sobrecarga de trabalho, falta de atenção, falta de recursos, estresse familiar, insatisfação no trabalho e quantitativo baixo de efetivo. As categorias encontradas durante a análise do questionário foram: Qualidade na assistência, segurança do paciente e erros de medicação. Conclusão: 0 estudo mostrou alguns pontos de fragilidade relacionado a assistência e segurança do paciente no preparo e administração de medicamentos devido ao quantitativo de atribuições atreladas ao técnico de enfermagem. A implantação de novas estratégias deverá ser adotada para que seja prestada uma assistência de excelência.
\end{abstract}

DESCRITORES: Enfermagem; Erros de Medicação; latrogenia; Cuidados de Enfermagem.

\section{ABSTRACT}

Objective: Identify in the nursing technician's perception which factors contribute to the iatrogenic act during drug preparation and administration. Method: Field research of qualitative approach, performed in the medical clinic sector of a private general hospital of Rio de Janeiro. Through a semi-structured questionnaire applied to 40 technical nursing professionals. Results: The most prevalent factors for inducing error in the preparation and administration of drugs most cited in the professionals' view are double hours, work overload, lack of attention, lack of resources, family stress, job dissatisfaction and low numbers of staff. The categories found during the analysis of the questionnaire were: Quality of care, patient safety and medication errors. Conclusion: The study showed some weaknesses related to patient care and safety in the preparation and administration of medications due to the number of attributions linked to the nursing technician. The implementation of new strategies must be adopted to provide excellent assistance.

KEYWORDS: Nursing; Medication Errors; latrogeny; Nursing Care.

\section{RESUMEN}

Objetivo: Identificar en la percepción del técnico de enfermería qué factores contribuyen al acto iatrogénico durante la preparación y administración del medicamento. Método: Investigación de campo de enfoque cualitativo, realizada en el sector de la clínica médica de un hospital general privado de Río de Janeiro. A través de un cuestionario semiestructurado aplicado a 40 profesionales de enfermería técnica. Resultados: Los factores más frecuentes para la inducción de errores en la preparación y administración de los medicamentos más citados en la opinión de los profesionales son: horas dobles, sobrecarga de trabajo, falta de atención, falta de recursos, estrés familiar, insatisfacción laboral y bajo número de personal. Las categorías encontradas durante el análisis del cuestionario fueron: calidad de la atención, seguridad del paciente y errores de medicación. Conclusión: El estudio mostró algunas debilidades relacionadas con la atención y seguridad del paciente en la preparación y administración de medicamentos debido a la cantidad de atribuciones vinculadas al técnico de enfermería. La implementación de nuevas estrategias debe ser adoptada para proporcionar una asistencia excelente. PALABRAS CLAVE: Enfermería; Errores de Medicación; latrogenia; Cuidados de Enfermería.

RECEBIDO EM: 10/11/2019 APROVADO EM: 11/11/2019

\section{Taiane dos Santos Vieira}

Enfermeira. Pós-graduada em Enfermagem na Alta Complexidade pela Universidade Castelo Branco. Rio de Janeiro - Rio de Janeiro. Brasil. 


\section{Diogo da Silva Lisboa}

Enfermeiro. Graduado pela Universidade Castelo Branco. Rio de Janeiro - Rio de Janeiro. Brasil.

\section{Bruno da Silva Lourenço}

Enfermeiro. Graduado pela EEAN/UFRJ. Especialista em Terapia Intensiva pela Escola de Enfermagem Anna Nery/EEAN/UFRJ. Docência do Ensino Superior Universidade Candido Mendes. Mestrado Enfermagem Psiquiátrica/EEAN/UFRJ. Enfermeiro do Setor de Hemodiálise do HFB. Professor Auxiliar Estácio de Sá. Rio de Janeiro. (RJ). Brasil.

\section{Leonardo Lima de Moraes dos Reis}

Enfermeiro Pós-graduado em Estratégia em Saúde da Família pela Universidade Castelo Branco. Docente da Escola Técnica Centro Rio - Rio de Janeiro - Rio de Janeiro. Brasil.

\section{Alexandro Alves Ribeiro}

Enfermeiro Graduado pela Universidade Federal do Rio de Janeiro. Docente na Universidade Estácio de Sá. Mestre em Saúde da família pela UNESA. Especialista em Saúde Coletiva pela Universidade Gama Filho. Rio de Janeiro - Rio de Janeiro. Brasil

\section{Susana Reis Ferreira}

Enfermeira Graduada pela FENF/UERJ. Rio de Janeiro. Brasil. Mestre e Doutoranda em Enfermagem pela FENF/UERJ. Rio de Janeiro - Rio de Janeiro. Brasil.

\section{Luciana Quagliane Ribeiro}

Enfermeira. Especialista em saúde da família. Rio de Janeiro - Rio de Janeiro. Brasil. Mestre em Saúde da família. Rio de Janeiro - Rio de Janeiro. Brasil. Bacharel em Direito. Rio de Janeiro. Brasil. Servidora do município do Rio de Janeiro. Docente e Coordenadora na Universidade Estácio de Sá. Rio de Janeiro. Brasil.

\section{INTRODUÇÃO}

A realidade vivenciada pelos profissionais da saúde, no que tange à administração de medicamentos e seu preparo, é a força motriz deste estudo. A demanda a ser atendida não coaduna com o efetivo, que por falta de profissionais, se desdobra para atender o máximo de pessoas no mínimo de tempo possível. Isso acaba por desencadear em erros no processo de preparo e administração de medicamentos onde, por vezes, não se dá apenas por falta de conhecimento, mas também, pelo entendimento de que o atendimento de todos os pacientes é igualmente importante $^{(1)}$. A categoria de enfermagem, na área de saúde, possui grande responsabilidade na última barreira do processo de administração de medicamentos, dito isso, sua atuação é irrefutável para evitar erros desse aspecto ${ }^{(2)}$.

A prática no preparo e administração de medicamentos requer conhecimento científico, de forma que se promova a segurança do paciente, pois quanto maior o conhecimento, menor será o índice de erros. Isso se configura, quer seja por vivência no ambiente de trabalho ou pela mídia, a atenção que expõe os erros da enfermagem referente ao preparo e administração de medicamentos, uma vez que podem acarretar em danos reversíveis ou até mesmo irreversíveis, como o óbito ${ }^{(3)}$.

O Código de Ética dos Profissionais de Enfermagem (CEPE), expresso pela Resolução COFEN n. ${ }^{\circ}$ 564/2017, refere que é direito do paciente a prestação de uma assistência clínica livre de danos decorrentes de negligência, imperícia ou imprudência, oferecendo ao trabalhador o direito de adiar atividades quando o local de trabalho não oferecer condições seguras para tal realização. A separação entre os termos negligência, imperícia e imprudência são bem pequenas e acabam por conduzir o profissional de enfermagem a comportamentos bem parecidos ${ }^{(4)}$.

Em relação aos eventos adversos referentes ao erro no preparo e administração de medicamentos, podemos destacar que são consideradas ocorrências evitáveis, podendo alterar a segurança microbiológica, que correspondia ao preparo antecipado, modificando a química de algumas medicações, influenciando no resultado terapêutico ${ }^{(5)}$.

Ressalta-se que é importante que a enfermagem agregue conhecimento para aprimorar diariamente a sua capacidade em preparar e administrar os medicamentos, contudo, não é essa a realidade cotidiana, pois ainda existem profissionais que não possuem capacitação adequada para a função. Torna-se relevante que haja uma valorização relacionada à importância da conscientização, onde todos da equipe de saúde devem, em senso comum, buscar por um melhor cuidado ao paciente, fazendo com que a equipe profissional encontre estratégias que reduzam ou eliminem esses eventos adversos ${ }^{(6)}$.

O processo de preparo e administração de medicamentos é de grande responsabilidade, pois tal atribuição requer conhecimento para relevância de seu desempenho por se tratar dos cuidados que serão prestados ao paciente hospitalizado ${ }^{(7)}$.

Tendo em vista que a administração de medicamentos, no contexto hospitalar, é uma das atribuições da equipe de enfermagem, é imperioso que todos os envolvidos se mantenham atentos ao mínimo de erro relacionado a medicamentos, contudo, o erro no preparo e administração envolve diversos fatores, podendo fugir do controle da enfermagem, por ser um procedimento que envolve outros profissionais da área da 
saúde, podendo ser ocasionado pela farmácia ou médicos que realizam a prescrição ${ }^{(2)}$.

Os profissionais de enfermagem, mesmo não sendo responsáveis pela prescrição de medicamentos, cabendo esta tarefa a equipe médica, devem conhecer todas as etapas envolvidas no processo, para que possa evitar prejuízos aos pacientes. Erros de medicações são evitáveis e estão relacionados às condutas dos profissionais, podendo também ser considerados erros previsíveis de medicações danosas ao paciente ${ }^{(8)}$.

A rotina transforma as atividades, que no início parecem complexas, em tarefas mais simples e objetivas. Com relação à preparação de medicamentos pela equipe de enfermagem, tais rotinas estão atreladas ao cotidiano do serviço, onde os conhecimentos técnicos vão sendo colocados em prática de modo a melhorar a eficiência do serviço ${ }^{(1,2)}$.

O propósito de entender o cotidiano dos profissionais técnicos de enfermagem gerou as inquietações ao realizar o presente estudo, uma vez que erros na administração e no preparo de medicamentos estão se tornando cada vez mais frequentes no ambiente de trabalho destes profissionais. O estudo também busca um entendimento sobre quais são os fatores que influenciam ou contribuem para a ocorrência de eventos adversos no preparo e administração de medicamentos no cotidiano destes profissionais ${ }^{(9)}$.

Desta forma, o estudo apresenta como questão de pesquisa: Quais os fatores extrínsecos e intrínsecos possibilitam atos iatrogênicos durante o preparo e administração de medicamentos? E ressalta-se que os objetivos do estudo são: levantar os fatores que possibilitam atos iatrogênicos no preparo e administração de medicamentos durante a assistência de enfermagem; identificar na, percepção do técnico de enfermagem, quais os fatores contribuem para o ato iatrogênico durante o preparo e administração de medicamentos; discutir a importância da prevenção dos fatores que contribuem para o erro no preparo e administração de medicamentos.

O estudo justifica-se uma vez que a rotina de um hospital pode ser agitada e estressante e a realidade revela que o número de efetivos não condiz com a somatória de pacientes que precisam de cuidados imediatos e de assistência sistematizada e cuidadosa. Desta forma, graves erros podem ser cometidos, se a eficiência for preterida em detrimento da qualidade. Visto o exposto, observou-se a importância de que os profissionais tenham plena ciência dos riscos e tenham conhecimento dos danos ocasionados no preparo e administração errada dos medicamentos, para que, de alguma forma, sejam minimizados os erros cometidos por eles e, ao mesmo tempo, sanem as dúvidas frequentes.

Com o intuito de contribuir de forma positiva para o ensino, o estudo realizou uma análise de quais os erros no preparo e administração de medicamentos vêm acontecendo com frequência, seja por falta de capacitação ou conhecimento próprio. Sendo assim, através desta pesquisa, o estudo fornece um material recente que possibilita aos profissionais acadêmicos de enfermagem a clareza dos danos e riscos ao paciente. Busca-se, então, proporcionar respostas à problemática no que se refere ao preparo e administração de medicamentos, conscientizando o profissional de enfermagem que erros são passíveis de prevenção e estimulá-los, através da pesquisa, que busquem e agreguem novos conhecimentos.

Considerando que a administração de medicamento é uma das maiores responsabilidades da enfermagem e que erros podem causar efeitos prejudiciais ao paciente com sérias consequências, é de extrema importância que os profissionais de enfermagem identifiquem os fatores de riscos que possam levar à ocorrência dos erros. A identificação e medidas que minimizem a sua ocorrência para melhorar a qualidade de assistência prestada à sua clientela são imprescindíveis. Desta forma, o presente estudo visa contribuir para a informação e direcionamento dos profissionais no planejamento da assistência, visando estratégias e melhorias para prevenir iatrogenias.

\section{METODOLOGIA}

Trata-se de uma pesquisa de campo com abordagem qualitativa de caráter descritivo, a abordagem qualitativa traz uma proxi- midade entre o sujeito e objeto, trazendo compreensão no campo da subjetividade e do simbolismo, fazendo com que as ações, os projetos, as estruturas e a relação se tornem significativas. $\mathrm{O}$ estudo de caráter qualitativo tem como princípio fundamental a compreensão e análise do empírico no seu ambiente, onde o pesquisador deve aprender a utilizar sua própria pessoa como instrumento de confiabilidade ${ }^{(10)}$.

A pesquisa foi realizada no setor de clínica médica, nas dependências de um hospital geral privado de médio porte, situado no bairro de Campo Grande, na Zona Oeste do Rio de Janeiro. Optou-se por esta unidade de saúde devido a um dos integrantes desta pesquisa ter fácil acesso aos entrevistados através de um vínculo empregatício com a instituição e poder, através da sua rotina diária, observar a conduta individualizada de cada um, onde foram selecionados como sujeito de pesquisa os profissionais técnicos de enfermagem de ambos os gêneros, masculino e feminino, plantonistas diurnos, tendo em vista que a disponibilidade imposta pela instituição para realização da pesquisa foi o sistema diurno, impossibilitando a coleta de dados dos funcionários do plantão noturno.

A coleta de dados transcorreu no período de março a maio de 2016, sendo utilizado um questionário semiestruturado com consentimento livre e esclarecido aplicado individualmente no local de trabalho dos entrevistados.

Como critério de inclusão, optou-se pelos profissionais técnicos de enfermagem do plantão diurno, pois utilizam dessa prática do preparo e administração de medicamentos com frequência e estão próximos ao cliente no decorrer de sua internação, necessitando de assistência, cuja qual é de responsabilidade e competência do técnico de enfermagem. Optou-se pelos profissionais no exercício da profissão atuantes acima de seis meses.

Utilizou-se como critério de exclusão, os profissionais graduados em enfermagem, pois ainda que fizessem uso desta prática, por diversas vezes estão realizando funções administrativas e burocráticas. Definiu-se como critério de exclusão o profissional da 
categoria "auxiliar de enfermagem", devido à atualização de conhecimento e aprimoramento da profissão, encontram-se em minoria das unidades de saúde. Não foram considerados os erros decorrentes de outros profissionais que constituem a equipe multiprofissional de saúde.

Após consultar diversos profissionais da área de enfermagem e professores com o mesmo tema de interesse, realizou-se a confecção de um instrumento de coleta de dados, onde foi validado por uma equipe de expertises. Foi realizado um teste piloto com um grupo de dez sujeitos de pesquisa para que, desta forma, se possa reestruturar tal instrumento de coleta de dados. Criou-se um questionário de pesquisa semiestruturado com uma pergunta objetiva e dez opções de resposta, abrangendo fatores extrínsecos e intrínsecos e duas perguntas discursivas, dando margem para o entrevistado expor sua opinião frente à questão em pauta. Optou-se por utilizar esse método com intuito de facilitar a abordagem, tornando-a rápida, com o objetivo de identificar quais fatores contribuem para o erro no preparo e administração de medicamentos durante a assistência de enfermagem.

O procedimento adotado para elaboração do instrumento de coleta de dados se deu pela realização de um questionário semiestruturado totalizando sete perguntas, sendo quatro perguntas relacionadas ao perfil do profissional técnico de enfermagem, com uma pergunta objetiva e duas perguntas discursivas dando margem para o entrevistado expor sua opinião frente à questão em pauta com intuito de inteirar-se dos fatores que contribuem para o erro no preparo e administração de medicamentos durante a assistência de enfermagem.

$\mathrm{O}$ estudo foi submetido à Plataforma Brasil para análise e posterior envio a um Comitê de Ética em Pesquisa (CEP), sendo aprovado pelo CAAE $n^{\circ}$ 60392416.0.0000.05279. Foi apresentado e explicado aos entrevistados o Termo de Consentimento Livre e Esclarecido (TCLE), garantindo-lhe o sigilo e o anonimato, contemplando a Resolução n. ${ }^{\circ} 466$, de 12 de dezembro de 2012, do Conselho Nacional de Saúde (CNS) e a
Resolução n. ${ }^{\circ}$ 510, de 7 de abril de 2016, que dispõem sobre as diretrizes e normas regulamentadoras das pesquisas envolvendo seres humanos, sendo solicitado após a leitura, permissão e assinatura do termo.

Os entrevistados foram identificados por iniciais, fato que contribui para o anonimato e segurança dos dados referentes aos entrevistados. Cabe ressaltar que os instrumentos serão armazenados por um período de cinco anos pelos pesquisadores e, após este período, serão incinerados.

\section{RESULTADOS E DISCUSSÃO}

Foi realizada uma análise com todas as respostas encontradas referentes aos 40 profissionais técnicos de enfermagem de um hospital privado na zona Oeste do Rio de Janeiro, buscando identificar os elementos constituintes do objeto elencado.

Os dados foram coletados por meio de questionário semiestruturado, com sete perguntas sobre a temática abordada, sendo estas: questão 01: Idade?, questão 02 : Gênero feminino ou masculino?, questão 03: Qual número de vínculos empregatícios?, questão 04: Qual tempo de profissão exercida como técnico de enfermagem?, questão 05: $\mathrm{O}$ que você entende como ato iatrogênico?, questão 06: Marque nas alternativas abaixo, de acordo com sua experiência profissional, os fatores que contribuem para o ato iatrogênico durante o preparo e administração de medicamentos e; por fim, questão 07: Em sua opinião, qual a importância em identificar e prevenir fatores que contribuem para a iatrogenia no preparo e administração de medicamentos?

Desta forma foi elaborado um quadro onde serão descritos de forma organizada e sucinta os dados apresentados. Fez-se necessária a categorização de uma questão discursiva apresentada no questionário de pesquisa.

Na modalidade da questão de número cinco (5) "O que você entende como ato iatrogênico?", cuja abordagem fora feita visando evidenciar o conhecimento dos profissionais técnicos de enfermagem sobre iatrogenia, optou-se por dividir as respostas segundo a obtenção de resultados diferenciados. Identificou-se que $35 \%$ dos entrevistados alegaram que a descrição de iatrogenia se dá por reaçôes adversas.

Ressalta-se que, em relação aos assuntos ligados à assistência de enfermagem, os erros de medicação têm se destacado nos manuais que tratam sobre a segurança do paciente. No entendimento técnico, erro de medicação é definido como qualquer evento, passível de prevenção, que cause ou induza o uso equivocado e inadequado de medicamentos; diversos fatores podem estar ligados a estes eventos ${ }^{(11)}$.

Com base nas respostas obtidas, $25 \%$ dos entrevistados relatou que iatrogenia significa ser negligente, agir com negligência, comprometer o cliente negligenciando a cura, a manutenção e a vida.

A enfermagem é imprescindível na prevenção e redução dos erros de medicação. É necessário que os enfermeiros estejam bastante atentos na identificação, comunicação e análise das possíveis falhas no sistema de medicação, como também na supervisão, planejamento de intervenções e políticas para o crescimento e benefício da segurança, seja na assistência de enfermagem seja na administração dos medicamentos ${ }^{(12)}$.

A seguir, representando $40 \%$ dos entrevistados, eles relatam que iatrogenia são condutas erradas na administração de medicamentos, visando a importância do aprendizado e o investimento no aperfeiçoamento técnico.

"[...] efeitos colaterais ou adversos a uma conduta do profissional, por falta de conbecimento técnico ou científico [...]" (D. C. S. S. no20).

"[...] consequência de um ato seu devido à falta de atualização de procedimentos [...]" (R.M. no21).

"[...] complicaçôes ao paciente devido a postura inadequada frente a conduta de um procedimento por falta de conbecimento e dominio do mesmo [...]" (R. M. no23).

Esse resultado mostra que a responsabilidade da enfermagem no cuidar é ampla, tonando-se suscetível a erros devido 
a falhas na execução de seus respectivos procedimentos.

É fundamental que todos os profissionais envolvidos no processo se sintam pessoalmente responsáveis em garantir a segurança do cliente e a qualidade do serviço. De igual modo, os administradores das instituições precisam garantir a análise das causas adversas, bem como a notificação das ocorrências, a fim de que as devidas melhorias sejam feitas ${ }^{(13)}$.

Como por exemplo: "paciente com uso contínuo de medicação em bomba infusora”. Se o profissional não obtiver conhecimento sobre o equipamento, pode acarretar danos ao paciente. Sendo assim, é importante a atualização dos profissionais técnicos através da educação continuada, que pode ser promovida pela própria instituição empregatícia.

Os erros de medicação podem ocasionar diversos agravantes à saúde, com importantes repercussões econômicas e sociais que atingem diretamente a vida dos pacientes bem como na dos profissionais de saúde e da própria instituição, além de prolongar o tempo de internação e afetar o tratamento ${ }^{(14)}$.

Neste sentido, visualiza-se que existem formas de prevenir erros na administração e preparo de medicamentos através da implementação de estratégias que visem e garantam a segurança do paciente. Existe uma grande fragilidade em todo o processo devido à superlotação de paciente e o baixo dimensionamento de profissionais de enfermagem, isso acarreta erros no processo, que podem ter iniciado na primeira etapa, através da confecção da prescrição ou nas etapas posteriores a esse processo.

Observada a dificuldade da rotina do técnico de enfermagem, em função das múltiplas responsabilidades, não cabe a estes equacionar todos os impasses, porém acabam solucionando. Com o objetivo de identificar, na percepção do técnico de enfermagem, quais os fatores contribuem para o erro no preparo de administração de medicamentos durante a assistência de enfermagem, foi elaborado um quadro onde foram analisadas as variações de respostas, como a seguir:

Entre os profissionais entrevistados na pesquisa, observou-se que 13 indivíduos concluíram que a dupla jornada de trabalho pode ser um fator contribuinte para o ato iatrogênico. Muitas das vezes os profissionais técnicos de enfermagem necessitam de vários vínculos empregatícios devido sua situação financeira ser prejudicada por causa de baixos salários, prejudicando a qualidade de vida. Sendo assim, assume essa dupla jornada de trabalho em diferentes turnos e instituições, com intuito de ter uma vida financeira estável.

Entender a complexidade do trabalho da enfermagem é uma forma de evitar os erros, uma vez que a assistência de enfermagem é dificultada pelas condições de trabalho, tendo como agravante a grande demanda de atendimento, sobrecarga de trabalho e remuneração insatisfatória ${ }^{(11,12)}$.

Dos 40 entrevistados, observou-se que 21 dos profissionais afirmam que sobrecarga de trabalho contribui para o ato iatrogênico. Sabemos que, para a realização de uma boa assistência de enfermagem, precisamos ter condições de trabalho que favoreçam a rotina diária que, por sinal, é bastante atordoada. As atividades exigem alto grau de organização, produção e pressão do tempo que gera uma sobrecarga de trabalho na execução das atividades diárias.

O provimento de pessoal, em quantitativo adequado, é de total responsabilidade da instituição de saúde, pois promove segurança às principais necessidades do paciente, de acordo com suas especificidades clínicas, evitando riscos recorrentes de incidentes que agravem o estado de saúde dos pacientes, assim como a qualidade de vida do trabalho dos profissionais de saúde ${ }^{(16)}$.

Para 32 entrevistados, os atos iatrogênicos se dão por falta de atenção em razão das muitas atribuições e responsabilidades da equipe de enfermagem. Sabemos que todo o processo para administração de medicamentos pode, muitas vezes, ser caracterizado como longo, pois uma série de outros fatores influencia até a conclusão do ato.

Conversas paralelas geram a falta de atenção durante o processo de preparo e administração de medicamentos, contribuindo para erros, pois merece atenção, sem perder o foco daquilo que está fazen-

Quadro 1. Distribuição dos fatores que contribuem para o ato iatrogênico, em uma amostra de 40 indivíduos. Rio de Janeiro, RJ, Brasil, 2016.

\begin{tabular}{|l|c|}
\hline ATO IATROGÊNICO & N \\
\hline Dupla jornada & 13 \\
\hline Sobrecarga de trabalho & 21 \\
\hline Falta de atenção & 32 \\
\hline Falta de recursos e materiais & 9 \\
\hline Estresse familiar & 4 \\
\hline Remuneração baixa & 1 \\
\hline Falta de motivação & 5 \\
\hline Insatisfação no trabalho & 3 \\
\hline Quantitativo baixo de efetivo para demanda elevada de paciente & 22 \\
\hline
\end{tabular}


do, evitando possíveis danos e ações prejudiciais ao paciente ${ }^{(15)}$.

Outro fator escolhido pelos entrevistados foi o da falta de recursos e materiais, sendo 09 os profissionais que optaram por essa alternativa que é um dos fatores mais relevantes, uma vez que sem material e recursos necessários, o profissional acaba realizando alguma conduta, onde, na maioria parte das vezes, a unidade não disponibiliza de material suficiente, o que acaba acarretando em complicações ao paciente.

Um dos principais erros está relacionado às normas de biossegurança, a falta de materiais e utilização inadequada de materiais. Com medidas de prevenção, promoveria a redução de contaminações e surgimento de patógenos resistentes, afetando as medidas terapêuticas adotadas para melhoria do cliente ${ }^{(7)}$.

Dentre os 40 profissionais entrevistados, 04 relataram que o fator contribuinte para $\mathrm{o}$ ato iatrogênico está relacionado ao estresse familiar. O estresse extra-hospitalar vivenciado por tais profissionais influencia na conduta no ambiente intra-hospitalar de forma negativa, podendo ocasionar em erros no preparo e na administração de medicamentos.

Com apenas 01 entrevistado optando pelo fator "remuneração baixa", o que se entende que nem todos os profissionais enxergam a remuneração como um fator relevante para contribuição de atos iatrogênicos, pode-se concluir que nem todos os profissionais entrevistados tiveram essa percepção como um fator relevante para o ato iatrogênico. O que nos causa inquietação, já que a baixa remuneração está associada à dupla jornada de trabalho.

Devido à baixa remuneração do corpo técnico de enfermagem, desperta no profissional uma busca por outros serviços, com esse cotidiano difícil, por vezes, sua remuneração não coaduna com sua qualificação, o que acaba por desencadear em estresses agravantes, dispersando a atenção em meio a rotina de trabalho ${ }^{(15)}$.

Para 05 dos entrevistados, a insatisfação no trabalho influencia para que atos iatrogênicos aconteçam. A ausência de estímulo com a equipe técnica de enfermagem,
A ausência de estímulo com a equipe técnica

de enfermagem, as cobranças exacerbadas

e o ambiente de

trabalho foram uns

dos argumentos

utilizados pelos

profissionais

entrevistados.

Observou-se a

necessidade do

incentivo das

lideranças, no que

diz respeito aos

critérios profissionais

e pessoais, com intuito

de motivá-los.

as cobranças exacerbadas e o ambiente de trabalho foram uns dos argumentos utilizados pelos profissionais entrevistados. Observou-se a necessidade do incentivo das lideranças, no que diz respeito aos critérios profissionais e pessoais, com intuito de motivá-los.

Para 03 profissionais entrevistados, o fator insatisfação no trabalho foi escolhido, uma vez que o profissional desgastado por conta das rotinas maçantes, quantitativo elevado de paciente, frustração pela falta de reconhecimento de suas lideranças, acaba por dar contribuição para que algum erro ocorra.

Dentro dos 40 entrevistados, 22 profissionais alegaram que fatores contribuintes para iatrogenia estão relacionados ao quantitativo baixo de efetivo para demanda elevada de pacientes, onde os profissionais se sobrecarregam e, por vezes, deixam de assistir com excelência algum paciente por falta de tempo.

As inúmeras atividades que são exercidas pela enfermagem requerem um elevado grau de organização. A elevada demanda de pacientes para cada profissional é um dos vários motivos contribuintes para o erro $\mathrm{e}$ agravo da saúde do próprio profissional ${ }^{(16)}$.

Com base na questão sete ${ }^{(7)}$ do questionário de pesquisa, que aborda a questão: "Em sua opinião, qual a importância em identificar e prevenir fatores que contribuem para a iatrogenia no preparo e administração de medicamentos?”. Realizamos a categorização desta questão com intuito de interpretar as respostas dadas pelos entrevistados. Os participantes tiveram diferentes formas de explicar a questão citada acima, ressaltaram que tais fatores podem estar ligados uns aos outros e, consequentemente, atingir a segurança do paciente. Dividiu-se, portanto, em três categorias: Qualidade na assistência, Segurança do paciente e Erros de medicação.

A Categoria 1, denominada de "Qualidade na assistência" foi construída a partir da experiência vivida pelos profissionais técnicos de enfermagem, onde trouxeram percepções acerca de suas realidades, decorrentes da qualidade prestada na assistência de enfermagem. Relatando suas preocupações no processo do cuidado. Dando assim, fundamento para resposta de 13 entrevistados que se basearam em qualidade na assistência, de acordo com as falas a seguir:

\footnotetext{
"[...] Para melhor atendimento ao paciente, de acordo com o tratamento e conduta mantida para que a resposta do mesmo seja eficaz [...]" (V.S. no38).
} 
"[...] Para contribuir para melhor assistência ao cliente, onde o mesmo terá um tratamento eficaz, favorecendo na sua recuperação [...]" ( $M$. G. A. n' 15 ).

\section{"[...] Identificando e prevenindo tais fatores, a tendência em promo- ver a qualidade na assistência au- menta [...]" (D. B. L. no 18).}

Priorizar a segurança do paciente, de forma que se dissemine por todos os serviços de saúde. Entende-se que qualquer erro ou falha é esperado em qualquer instituição, sendo, assim, necessárias estratégias para prevenção de tais erros, tendo em vista o incentivo à atualização e capacitação do profissional de enfermagem ${ }^{(17)}$.

A categoria 2, sobre a "Segurança do Paciente" remete que de acordo com falas nessa categoria, os técnicos de enfermagem atribuem a importância de prevenir iatrogenias no preparo e administração de medicamentos a uma forma de controle de possíveis complicações, garantindo a integridade e segurança do paciente. Obtendo 11 respostas voltadas para segurança do paciente.

\section{“[...] Para evitar que o tratamento implantado perca o resultado espera- do, evitando causar danos ao pacien- te, o assegurando de prejuizos duran- te a assistência [...]" (Q. V.S. no 16).}

\section{"[...] Identificar e prevenir esses atos são um dos meios para o qual o pa- ciente não tenha nenhuma reper- cussão direta na sua vida e saúde, ou seja, nenhum resultado danoso causado por erros [...]" (M. J. no 08). \\ “[...] Para segurança ao cliente, pro- vendo uma assistência livre de da- nos [...]" (C.M. no 5).}

A segurança do paciente entende-se como a prevenção de erros no cuidado dispensado ao cliente, bem como de danos causados por tais erros. Dessa forma, os erros cometidos pela equipe de enfermagem, resultante ou não de uma ação intencional causado por alguma falha ou problema, durante a assistência ao paciente, bem como preparo e administração de medicamentos, comprometem a segurança dos pacientes ${ }^{(16)}$.

$\mathrm{Na}$ categoria 3, que versa sobre "Erros de Medicação", 16 entrevistados acharam relevante a prevenção de erros de medicação na assistência prestada ao paciente, evitando complicações causadas pelo profissional, pois, desta forma, acredita-se que iatrogenias podem ser desencadeadas por um ato prejudicial.

"[...] Evitando erros e possiveis complicações futuras ao cliente [...]" (G.S. $n^{\circ} 4$ ).

\section{“[...] É importante para garantir ao cliente um tratamento digno que seja livre de erros, com segurança, de forma adequada, para se alcançar os resultados esperados de um trata- mento clínico [...]" (A. A. no 11).}

Erros de medicação foram um dos fatores citados pelos entrevistados, sendo que esses participantes destacam que esse fator é o de maior prevalência para ocorrência de iatrogenias medicamentosas, fica evidente que, para o preparo e administração corretas são necessários métodos de barreira, pois o erro também pode ser iniciado tanto pelo médico quanto farmacêutico e assim sucessivamente.

Com o despreparo e desatualização de conhecimento dos profissionais, pode acarretar erros no preparo e administração de medicamentos. Neste sentido, uma forma de prevenir tais fatores seria a implementação de mecanismos de segurança, tirando o foco da punição pelo erro ocorrido e direcionando a atenção para formas de melhoria e educação continuada ${ }^{(18,19)}$.

\section{CONCLUSÃO}

O ambiente hospitalar, mesmo frente às adversidades inerentes ao serviço que lhe é peculiar, não pode ser eximido de prestar um cuidado de qualidade, quer seja a mais basilar ou de maior complexidade.
Isso implica em urgência cuidadosa, em medicação fiscalizada e em coerência organizacional. A desatenção em qualquer um destes pontos pode ser implacavelmente ruim para o cliente e, por fim, para a instituição. É imprescindível que haja um controle de qualidade na averiguação fidedigna em relação ao preparo e administração dos medicamentos. Com o objetivo de exaurir os erros, a prevenção e a precaução devem ser pontos cardeais.

$\mathrm{O}$ estudo mostrou alguns pontos de fragilidade no que diz respeito à assistência e segurança do paciente com relação ao preparo e administração de medicamentos devido ao quantitativo de atribuições atreladas ao técnico de enfermagem. Medidas de prevenção e implantação de novas estratégias deverão ser adotadas para que seja prestada uma assistência de excelência.

Os resultados obtidos na pesquisa, uma vez que, de acordo com os profissionais participantes dessa pesquisa, o diagnóstico do problema varia, sendo causado por motivos multifatoriais, sendo os mais pontuais: a falta de atenção, o quantitativo baixo de efetivo para demanda elevada de paciente e a sobrecarga de trabalho. Em tese, entende-se que são problemas interligados, uma vez que um acarreta outro.

A implantação de medidas preventivas, além de fundamental, é imperativa. A pesquisa não visa ser única, mas somar à relevância a fim de que o trabalho profissional seja cada vez mais qualitativo. É de suma importância que haja notificação dos erros para que seja feito um levantamento minucioso de seu quantitativo, dando margem para a criação de medidas de prevenção, com isso, identificando as dificuldades dos profissionais, pois conhecer todas as etapas desse processo é fundamental.

Conquanto as deficiências sejam grandes, o presente estudo contribuirá de forma positiva a fim de alertar o profissional quanto à importância do entendimento acerca dos erros durante o preparo e administração de medicamentos. Assim sendo, a assistência prestada será segura e de confiança. 


\section{AGRADECIMENTO}

Ao Professor Fabrício Fernandes Pinto "in memorian" pela contribuição na elaboração deste estudo, mas que infelizmente não pôde ver a concretização desta etapa.
Uma pesquisa árdua, com muitos envolvidos, que atravessou momentos desafiadores, mas que seu conhecimento foi fundamental para superar esses obstáculos. Será sempre recordado por seu exemplo como Enfermeiro, Professor e grande homem que foi.
Sua família, amigos e colegas de profissão sentem orgulho de terem estado com você nesse curto tempo. A saudade aperta, mas o fruto do seu trabalho e nossas lembranças nos fazem seguir".

\section{REFERÊNCIAS}

1. Gomes ATL, et al. Erros na administração de medicamentos: Evidências e implicações na segurança do paciente. Cogitare Enfermagem [Internet]. 2016 [acesso em 01 dez 2019]; 21(3): 01-11. DOI: https://doi.org/10.5380/ce.v21i3.44472.

2. Forte ECN, et al. Errores de Enfermería: Qué está en estudio. Texto \& contexto enfermagem [Internet]. 2017 [acesso em 01 dez 2019]; 26(2). DOI: https://doi.org/10.1590/010407072017001400016.

3. Ministério da Saúde, Agência Nacional de Vigilância Sanitária (BR). Assistência segura: uma reflexão teórica aplicada à prática. Série Segurança do Paciente e Qualidade em Serviços de Saúde [Internet]. Anvisa; 2017 [acesso em 01 dez 2019]. Disponivel em: https://www20.anvisa.gov.br/segurancadopaciente/index. php/publicacoes/item/caderno-1-assistencia-segura-uma-reflexao-teorica-aplicada-a-pratica.

4. Conselho Federal de Enfermagem (BR). Resolução COFEN n. ${ }^{\circ}$ 564/2017, de 06 de dezembro de 2017, que aprova o novo Código de Ética dos profissionais de Enfermagem [Internet]. Brasília (DF): COFEN, 2017 [acesso em 20 set 2019]. Disponivel em: http://www.cofen.gov.br/publicado-novo-codigo-de-etica-da-enfermagem-brasileira_59153.html.

5. Alsulami Z, Conroy S, Choonara I. Medication errors in the Middle East countries: a systematic review of the literature. Eur J Clin Pharmacol [Internet]. 2013 [acesso em 01 dez 2019]; 69:995-1008. DOl: https://doi.org/10.1007/s00228-0121435-y.

6. Leitão IMTA, et al. Análise da comunicação de eventos adversos na perspectiva de enfermeiros assistenciais. Rev Rene [Internet]. 2013 [acesso em 01 dez 2019]; 4(3). 14(6):1073-83. DOI: http://dx.doi.org/10.15253/rev\%20rene.v14i6.3713.

7. Menezes Ferreira MM, Barberino Jacobina FM, Silva Alves FD. O profissional de enfermagem e a administração segura de medicamentos. Revista Enfermagem Contemporânea. Escola Bahiana de Medicina e Saúde Pública [Internet]. 2014 [acesso em 01 dez 2019]; 3(1):61-69. DOI: http://dx.doi.org/10.17267/ 2317-3378rec.v3i1.208.

8. Pichler RF, et al. Erros de medicação: análise ergonômica de utensílios da sala de medicação em ambiente hospitalar. Cadernos saúde coletiva [Internet]. 2014 [acesso em 01 dez 2019]; 22(4):365-371. DOI: https://doi.org/10.1590/ $1414-462 \times 201400040004$.

9. Andrade PDP, Amaral TDS, Omizzolo JAE. Segurança do paciente: administração segura de medicamentos. Inova Saúde. Fundacao Educacional de Criciuma- FUCRI [Internet]. 2015 Dec [acesso em $01 \mathrm{dez}$ 2019]; 17;4(2):45. DOI: https://doi. org/10.18616/is.v4i2.1948.45-60.

10. Minayo, MCS. Análise qualitativa: teoria, passos e fidedig- nidade. Ciência \& saúde coletiva [Internet]. 2012 [acesso em 01 dez 2019]; 17(3):621-626. DOI: http://dx.doi.org/10.1590/ S1413.

11. Forte ECN, Machado FL, Pires DEP. A relação da enfermagem com os erros de medicação: uma revisão integrativa. Cogitare Enfermagem [Internet]. 2016 [acesso em 01 dez 2019]; 21(esp):01-10. DOI: https://doi.org/10.5380/ce.v21i5.43324.

12. Souta MM, et al. Sistema de Medicação: Análise das ações dos profissionais em Unidades de Internação Psiquiátrica. Texto \& contexto enfermagem [Internet]. 2016 [acesso em $01 \mathrm{dez}$ 2019]; 25(4):e0170015. DOI: https://doi.org/10.1590/010407072016000170015

13. Bohomol E. Erros de medicação: estudo descritivo das classes dos medicamentos e medicamentos de alta vigilância. Escola Anna Nery [Internet] 2014 [acesso $01 \mathrm{dez} 2019$ ]; 18(2):311316. DOI: https://doi.org/10.5935/1414-8145.20140045.

14. Volpe CRG, et al. Risk factors for medication errors in the electronic and manual prescription. Revista latino-americana de enfermagem [Internet]. 2016 [acesso em 01 dez 2019]; 24:e2742. DOI: https://doi.org/10.1590/15188345.0642 .2742 .

15. Mendonça VF. A Relação entre o Sedentarismo, Sobrepeso e Obesidade com as Doenças Cardiovasculares em Jovens Adultos: uma Revisão da Literatura. Revista brasileira de crescimento e desenvolvimento humano [Internet]. 2016 [acesso em 01 dez 2019]; 4(1): 79-90. DOI: https://doi.org/10.18316/23178582.16.21.

16. Lima MB, et al. Agentes estressores em trabalhadores de enfermagem com dupla ou mais jornada de trabalho. $\mathrm{R}$ p online cuid é fund [Internet]. $2013 \mathrm{jan} . / \mathrm{mar}$ [acesso em 01 dez 2019]; 5(1):3259-66. DOI: https://doi.org/10.9789/ 2175-5361.2013v5n1p3259.

17. Oliveira RM, et al. Estratégias para promover segurança do paciente: da identificação dos riscos às práticas baseadas em evidências. Escola Anna Nery [Internet]. 2014 [acesso em $01 \mathrm{dez}$ 2019]; 18(1):122-129. DOl: http://dx.doi.org/10.5935/14148145.20140018

18. Paim RSP, Bellaver DC, Belmonte J, Azeredo JC. Erros de medicação e segurança do paciente: uma revisão integrativa da literatura. Revista eletrônica Gestão \& Saúde [Internet]. 2016 [acesso em 01 dez 2019]; 7(3):1256-70. DOI: http://dx.doi. org/10.18673/gs.v7i3.21994.

19. Pustiglione M. A segurança (e saúde) do trabalhador da saúde e a segurança do paciente: uma análise do impacto das ações de segurança e saúde no trabalho em serviços de saúde. Revista de Administração em Saúde [Internet]. 2017 Jan [acesso em 01 dez 2019]; 17(66). DOI: https://doi.org/10.23973/ras.66.15. 